\title{
Convergence Analysis of shifted Fourth kind Chebyshev Wavelets
}

\author{
Suha N. Shihab ${ }^{(1)}$, Mohammed Abdulhadi Sarhan ${ }^{(2)}$ \\ ${ }^{(1)}$ Applied Science Department, University of Technology \\ ${ }^{(2)}$ College of Science , Al-mustansiriahUniversity
}

Abstract: The aim of this paper is to state and prove the uniform convergence theorem and accuracy estimation for shifted fourth kind Chebyshev wavelets.

Keywords: fourth kind Chebyshev polynomials, Chebyshev wavelets, uniform convergence.

\section{Introduction}

Wavelets theory is a relatively new and an emerging area in mathematical research. Wavelets Permit the accurate representation of a variety of functions and operators, and establish a connection with fast numerical algorithms.

There are several advantages of using Chebyshev wavelets approximation, therefore Chebyshev wavelets have become increasingly crucial in numerical analysis. It is well known that there are four kinds of Chebyshev wavelets. In the literature, there is a great concentration on the first and second kinds of Chebyshev wavelets $\Psi_{n}^{\lambda}(x)$ and $\Psi_{n}^{2}(x)$ respectively and their various use sapplications. A numerical method for onedimensional Bratu's problem based on Chebyshev wavelets of the first kind was presented in [1], FariborziAraghi [2] proposed a method to approximate the solution of a linear Fredholm integro-differential equation via Chebyshev wavelets of the first kind. Ali [3] applied Chebyshev wavelets method for delay differential equations. See [4-8] for other works.

Usually Chebyshev wavelets of third and fourth kinds are known less than first and second kinds in the literature. Third kind Chebyshev wavelets were studied [9-11]. We therefore intend in this work to introduce shifted Chebyshev wavelets of fourth kind together with convergence analysis and its error estimation.

\section{Fourth Kind Chebyshev Polynomials:}

Chebyshev polynomials of forth kind $W_{n}(x)$ are polynomials of degree $\mathrm{n}$ in $\mathrm{x}$, and defined by $[12,13]$

where

$$
W_{n}(x)=\frac{\sin (n+(1 / 2) \theta)}{\sin (\theta / 2)}
$$

The polynomials $W_{n}(x)$ are orthogonal on $(-1,1)$, that is

$$
\int_{-1}^{1} w(x) W_{n}(x) W_{m}(x) \mathrm{dx}= \begin{cases}\pi & \mathrm{n}=\mathrm{m} \\ 0 & \mathrm{n} \neq \mathrm{m}\end{cases}
$$

where $w(x)=\sqrt{\frac{1-x}{1+x}}$

and they may be generated by using the recurrence relation

$W_{n}(x)=2 x W_{n-1}(x)-W_{n-2}(x) \quad \mathrm{n}=2,3, \ldots$

with the initial values $W_{0}(x)=1, W_{n}(x)=2 x+1$

on the other hand, since the zeros of the sequences are as [15]

The polynomials are decomposed in the form

$$
W_{n}(x)=0 \rightarrow x_{k}=\cos \frac{2 k \pi}{2 n+1}, k=1,2, \ldots, n
$$

$$
W_{n}(x)=\sum_{k=0}^{n}(-1)^{k}\left(\begin{array}{c}
2 n+1 \\
2 k+1
\end{array}\right)\left(\frac{1-x}{2}\right)^{k}\left(\frac{1+x}{2}\right)^{n-k}
$$

or

$$
W_{n}(x)=2^{n} \pi_{k=1}^{n}\left(x-\cos \frac{2 k \pi}{2 n+1}\right)
$$

The shifted Chebyshev polynomials of the forth kind one defined on $[0,1]$, as

$$
W_{n}^{*}(x)=W_{n}(2 x-1)
$$

The orthogonality relation of $w_{n}^{*}(t)$ is given by

$$
\int_{0}^{1} w^{*}(t) W_{n}^{*}(t) W_{m}^{*}(t) d t= \begin{cases}\frac{\pi}{2} & m=n \\ 0 & m \neq n\end{cases}
$$


where $w^{*}=\sqrt{\frac{1-t}{t}}$

and

with $W_{0}^{*}=1, W_{1}^{*}=2 x+1$

$$
W_{n+1}^{*}=2(2 x-1) W_{n}^{*}(x)-W_{n-1}^{*}(x)
$$

\section{Fourth Kind Chebyshev Wavelets}

Wavelets constitute a family of functions constructed from dilation and translation of single function called the mother wavelets when the dilation parameter a and the we have the following family of continuous wavelets

$$
\Psi_{a, b}(t)=|a|^{-1 / 2} \Psi\left(\frac{t-b}{a}\right) \quad a, b \in R, a \neq 0
$$

Chebyshev wavelets of the fourth kind $\Psi_{n, m}^{4}(t)=\Psi(k, n, m, t)$ have four arguments $\mathrm{n}$ argument, $\mathrm{k}$ can assume any positive integer, $\mathrm{m}$ is the order for Chebyshev polynomials $w_{n}(t)$ and $\mathrm{t}$ is the normalized time, they are defined on are interval $[0,1]$ by

$$
\Psi_{n, m}^{4}(t)=\left\{\begin{array}{cc}
2^{k+1 / 2} & P_{m}\left(2^{k} t-2 n+1\right) \frac{n-1}{2^{k-1}} \leq t<\frac{n}{2^{k-1}} \\
0 & \text { otherwise }
\end{array}\right.
$$

where $P_{m}(t) \frac{1}{\sqrt{\pi}} W_{m}(t) \quad$ and $\mathrm{m}=0,1, \ldots, \mathrm{M} \quad \mathrm{n}=1,2, \ldots, 2^{k-1}$

we should note that in dealing with Chebyshev wavelets the weight function $w(x)$ have to be dilated and translated as

$w_{n}(x)=w\left(2^{k} t-2 n+1\right)$.

\section{Convergene Analysis for Chebyshev Wavelets of Fourth Kind.}

A function $f(t)$ defined over $[0,1]$ may be expanded as:

$$
f(t)=\sum_{n=1}^{\infty} \sum_{m=0}^{\infty} f_{n m} \Psi_{n m}^{4}(t)
$$

where

$$
f_{n m}=\left(f(t), \Psi_{n m}^{4}(t)\right)
$$

In eq.(6).(.,.) denotes the inner product with weight function $w_{n}(t)$.

If the infinite series in eq.(5) is trancated then eq.(5) can be written as:

$$
\begin{gathered}
f(t) \simeq f_{2^{k} M-1}(t)=\sum_{n=1}^{2^{k}} \sum_{m=0}^{M-1} f_{n m} \Psi_{n m}^{A} \\
=F^{T} \Psi(t)
\end{gathered}
$$

where $\mathrm{F}$ and $\Psi(t)$ are $2^{k} M \times 1$ matrices given by

$$
\begin{gathered}
F=\left[f_{10}, f_{11}, \ldots, f_{1 M}, f_{20}, \ldots, f_{2 M-1}, \ldots, f_{2^{k} 0}, \ldots, f_{2^{k} M-1}\right]^{T} \\
\Psi(\mathrm{t})=\left[\Psi_{10}^{4}(t), \Psi_{11}^{A}(t), \ldots, \Psi_{1 M-1}^{4}(t), \Psi_{20}^{A}(t), \ldots, \Psi_{2 M-1}^{4}(t), \Psi_{2^{k}}^{4}(t), \ldots, \Psi_{2^{k} M-1}^{4}(t)\right]^{T}
\end{gathered}
$$

\section{Theorem(1): (Convergence Analysis theorem)}

Assume that a function $\mathrm{f}(\mathrm{t}) \in L_{w^{*}}^{2}[0,1], w^{*}=\sqrt{\frac{1-t}{t}}$ with $\left|f^{\prime \prime}(t)\right| \leq L$, can be expanded as infinite series of fourth kind chebyshev wavelets, then the series converges uniformly to $\mathrm{f}(\mathrm{t})$.

Proof: since $f_{n m}=\left(f(t), \Psi_{n m}^{4}(t)\right)$

then

$$
\begin{aligned}
f_{n m} & =\int_{0}^{1} f(t) \Psi_{n m}^{4}(t) w_{n}(t) d t \\
& =\int_{n-1}^{n} 2_{2^{k-1}} \quad \frac{2^{\frac{k+1}{2}}}{\sqrt{\pi}} f(t) W_{m}\left(2^{k} t-2 n+1\right) w\left(2^{k} t-2 n+1\right) d t
\end{aligned}
$$

If we make use of the substitution $2^{k} t-2 n+1=\cos \theta$ in (10), yields

$f_{n m}=\frac{1}{2^{\frac{k+1}{2}} \sqrt{\pi}} \int_{0}^{\pi} f\left(\frac{\cos \theta+2 n-1}{2^{k}}\right) 2 \sin \left(\left(m+\frac{1}{2}\right) \theta\right) \sin \frac{1}{2} \theta d \theta$

By using the integration by parts,

Then eq.(11) becomes 


$$
\begin{gathered}
f_{n m}=\frac{1}{2^{\frac{k+1}{2} \sqrt{\pi}}}\left[\left.\left[f\left(\frac{\cos \theta+2 n-1}{2^{k}}\right)\left(\frac{\sin m \theta}{m}-\frac{\sin (m+1) \theta}{m+1}\right)\right]\right|_{0} ^{\pi}\right. \\
\left.+2^{-k} \int_{0}^{\pi} f^{\prime}\left(\frac{\cos \theta+2 n-1}{2^{k}}\right) \sin \theta\left(\frac{\sin m \theta}{m}-\frac{\sin (m+1) \theta}{m+1}\right)\right] d \theta \\
f_{n m}=\frac{1}{2 m 2^{\frac{3 k+1}{2}} \sqrt{\pi}} \int_{0}^{\pi} f^{\prime}\left(\frac{\cos \theta+2 n-1}{2^{k}}\right) \sin \theta \sin m \theta d \theta-\frac{1}{2(m+1) 2^{\frac{3 k+1}{2}} \sqrt{\pi}} \int_{0}^{\pi} f^{\prime}\left(\frac{\cos \theta+2 n-1}{2^{k}}\right) \sin \theta \sin (m+1) \theta d \theta
\end{gathered}
$$

After performing integration by parts again yields,

$$
\begin{gathered}
f_{n m}=\frac{1}{2^{2} m 2^{\frac{4 k+1}{2} \sqrt{\pi}}} \int_{0}^{\pi} f^{\prime \prime}\left(\frac{\cos \theta+2 n-1}{2^{k}}\right)\left[\frac{\cos (m-2) \theta-\cos m \theta}{m-1}-\frac{\cos m \theta-\cos (m+2) \theta}{m+1}\right] d \theta \\
-\frac{1}{2^{2}(m+1) 2^{\frac{4 k+1}{2}} \sqrt{\pi}} \int_{0}^{\pi} f^{\prime \prime}\left(\frac{\cos \theta+2 n-1}{2^{k}}\right)\left[\frac{\cos (m-1) \theta-\cos (m+1) \theta}{m}\right. \\
\left.-\frac{\cos (m+1) \theta-\cos (m+3) \theta}{m+2}\right] d \theta
\end{gathered}
$$

Consider:

$$
\begin{gathered}
\left|\int_{0}^{\pi} f^{\prime \prime}\left(\frac{\cos \theta+2 n-1}{2^{k}}\right)\left[\frac{\cos (m-2) \theta-\cos m \theta}{m-1}-\frac{\cos m \theta-\cos (m+2) \theta}{m+1}\right] d \theta\right|^{2} \\
=\left|\int_{0}^{\pi} f^{\prime \prime}\left(\frac{\cos \theta+2 n-1}{2^{k}}\right)\left[\frac{\cos (m-2) \theta-\cos m \theta}{m-1}-\frac{\cos m \theta-\cos (m+2) \theta}{m+1}\right] d \theta\right|^{2} \\
\leq \int_{0}^{\pi}\left|f^{\prime \prime}\left(\frac{\cos \theta+2 n-1}{2^{k}}\right)\right|^{2} d \theta \times \int_{0}^{\pi}\left|\frac{(m-1) \cos (m+2) \theta-2 m \cos m \theta+(m+1) \cos (m-2) \theta}{(m-1)(m+1)}\right|^{2} d \theta \\
<\pi L^{2} \int_{0}^{\pi} \frac{(m-1)^{2} \cos ^{2}(m+2) \theta+4 m^{2} \cos ^{2} m \theta+(m+1)^{2} \cos ^{2}(m-1)}{(m-1)^{2}(m+1)^{2}} d \theta \\
=\frac{\pi L^{2}}{(m-1)^{2}(m+1)^{2}}\left[\frac{\pi}{2}(m-1)^{2}+\frac{\pi}{2} 4 m^{2}+\frac{\pi}{2}(m+1)^{2}\right] \\
=\frac{\pi^{2} L^{2}}{(m-1)^{2}(m+1)^{2}}\left[3 m^{2}+1\right]
\end{gathered}
$$

Thus, we get

$\left|\int_{0}^{\pi} f^{\prime \prime}\left(\frac{\cos \theta+2 n-1}{2^{k}}\right)\left[\frac{\cos (m-2) \theta-\cos m \theta}{m-1}-\frac{\cos m \theta-\cos (m+2) \theta}{m+1}\right] d \theta\right|<\frac{\pi L\left(3 m^{2}+1\right)^{1 / 2}}{(m-1)(m+1)}$

Similarly,

$$
\begin{gathered}
\left|\int_{0}^{\pi} f^{\prime \prime}\left(\frac{\cos \theta+2 n-1}{2^{k}}\right)\left[\frac{\cos (m-1) \theta-\cos (m+1) \theta}{m}-\frac{\cos (m+1) \theta-\cos (m+3) \theta}{m+2}\right] d \theta\right|^{2} \\
=\left|\int_{0}^{\pi} f^{\prime \prime}\left(\frac{\cos \theta+2 n-1}{2^{k}}\right) \times \frac{m \cos (m+3) \theta-(2 m-2) \cos (m+1) \theta+(m+2 \theta) \cos (m-1) \theta}{m(m+2)}\right|^{2} \\
\leq \int_{0}^{\pi}\left|f^{\prime \prime}\left(\frac{\cos \theta+2 n-1}{2^{k}}\right)\right|^{2} d \theta \times \int_{0}^{\pi}\left|\frac{m \cos (m+3) \theta-(2 m-2) \cos (m+1) \theta+(m+2 \theta) \cos (m-1) \theta}{m(m+2)}\right|^{2} \\
<\pi L^{2} \int_{0}^{\pi} \frac{m^{2} \cos ^{2}(m+3) \theta+(2 m-2)^{2} \cos ^{2}(m+1) \theta+(m+2)^{2} \cos ^{2}(m-1) \theta}{m^{2}(m+2)^{2}} \\
=\frac{\pi L^{2}}{m^{2}(m+2)^{2}}\left[m^{2} \frac{\pi}{2}+\frac{\pi}{2}(2 m-2)^{2}+\frac{\pi}{2}(m+2)^{2}\right]
\end{gathered}
$$

Thus we get

$$
\begin{aligned}
& \left|\int_{0}^{\pi} f^{\prime \prime}\left(\frac{\cos \theta+2 n-1}{2^{k}}\right)\left[\frac{\cos (m-1) \theta-\cos (m+1) \theta}{m}-\frac{\cos (m+1) \theta-\cos (m+3) \theta}{m+2}\right]\right| d \theta \\
& <\frac{\pi L}{m(m+2)}\left(3 m^{2}-2 m+4\right)^{1 / 2}
\end{aligned}
$$

Using eqs.(14)and(15), one can get

$$
\left|f_{n m}\right|<2^{-\frac{4 k+1}{2}-2} \pi^{1 / 2}\left(\frac{\pi L\left(3 m^{2}+1\right)^{1 / 2}}{m(m-1)(m+1)}-\frac{\pi L\left(3 m^{2}-2 m+4\right)^{1 / 2}}{m(m+1)(m+2)}\right)
$$


Finally, since $n \leq 2^{k}-1$, then

$$
\begin{aligned}
\left|f_{n m}\right|< & \frac{2^{\frac{-5 k}{2}-2} \pi^{\frac{1}{2}} L}{m}\left(\frac{2 m}{(m-1)^{2}}\right) \\
& =\frac{22^{\frac{-5 k}{2}-2} \pi^{\frac{1}{2}} L}{(m-1)^{2}}
\end{aligned}
$$

$\left|f_{n m}\right|<\frac{\pi^{\frac{3}{2}} L(n+1)^{\frac{-5}{2}}}{2(m-1)^{2}}$

\section{Accuracy Estimation of $\Psi_{n m}^{4}(x)$}

If the function $\mathrm{f}(\mathrm{x})$ is expanded in terms of fourth kind Chebyshev wavelets,

$$
f(x)=\sum_{n=1}^{\infty} \sum_{m=0}^{\infty} f_{n m} \Psi_{n m}^{A}(x)
$$

It is not possible to perform computation an infinite number of terms, therefore we must truncate the series in (17). In place of (17), we take

so that

$$
f_{M}(x)=\sum_{n=1}^{2^{k-1}} \sum_{m=0}^{M-1} f_{n m} \Psi_{n m}^{A}(x)
$$

or

$$
\begin{aligned}
& f(x)=f_{M}(x)+\sum_{n=2^{k-1}+1}^{\infty} \sum_{m=M}^{\infty} f_{n m} \Psi_{n m}^{A}(x) \\
& f(x)-f_{M}(x)=r(x)
\end{aligned}
$$

where $\mathrm{r}(\mathrm{x})$ is the residual function

$$
r(x)=\sum_{n=2^{k-1}+1}^{\infty} \sum_{m=M}^{\infty} f_{n m} \Psi_{n m}^{A}(x)
$$

we must select coefficients in eqs.(18) and (19) such that the norm of the residual function $\|r(x)\|$ is less than some convergence criterion $\in$, that is

for all $\mathrm{M}$ greater than some value $M_{0}$.

$$
\left(\int_{0}^{1}\left(f(x)-f_{M}\right)^{2} w_{n}(x) d x\right)^{\frac{1}{2}}<\epsilon
$$

\section{Theorem (2)}

Let $\mathrm{f}(\mathrm{x})$ be a continuous function defined on $[0,1)$, and $\left|f^{\prime \prime}(x)\right|<L$, then we have the following accuracy estimation

where

$$
c_{k, M}<\frac{\pi^{2} L}{2} \sum_{n-2^{k-1}+1}^{\infty} \sum_{m=M}^{\infty} \frac{1}{(n+1)^{5}} \frac{1}{(m-1)^{4}}
$$

\section{Proof}

$$
c_{k, M}=\left(\int_{0}^{1}(r(x))^{2} w_{n}(x) d x\right)^{\frac{1}{2}}
$$

Since $\quad c_{k, M}=\left(\int_{0}^{1}(r(x))^{2} w_{n}(x) d x\right)^{\frac{1}{2}}$

Then

$$
\begin{gathered}
c_{k M}^{2}=\int_{0}^{1}(r(x))^{2} w_{n}(x) d x=\int_{0}^{1} \sum_{n=2^{k-1}+1}^{\infty} \sum_{m=M}^{\infty} f_{n m}^{2}\left(\Psi_{n m}^{4}(x)\right)^{2} w_{n}(x) d x \\
=\sum_{n=2^{k-1}+1}^{\infty} \sum_{m=M}^{\infty} f_{n m}^{2} \int_{0}^{1}\left(\Psi_{n m}^{4}(x)\right)^{2} w_{n}(x) d x \\
=\sum_{n=2^{k-1}+1}^{\infty} \sum_{m=M}^{\infty} f_{n m}^{2}\left(2^{\frac{k}{2}}\right)^{2} \int_{\frac{n-1}{2^{k-1}}}^{\frac{n}{2^{k-1}}} P_{m}\left(2^{k} x-2 n+1\right)^{2}\left(\frac{1-\left(2^{k} x-2 n+1\right)}{1+\left(2^{k} x-2 n+1\right)}\right)^{\frac{1}{2}} d x
\end{gathered}
$$

Let $t=2^{k} x-2 n+1$ then $d t=2^{k} d x$

Therefor

$$
c_{k M}^{2}=\sum_{n=2^{k-1}+1}^{\infty} \sum_{m=M}^{\infty} f_{n m}^{2} 2^{-k} 2^{k} \int_{-1}^{1} W_{m}^{2}\left(\frac{1-t}{1+t}\right)^{\frac{1}{2}} d t
$$


we have

$$
\int_{-1}^{1} W_{m}^{2}\left(\frac{1-t}{1+t}\right)^{\frac{1}{2}} d t=\pi
$$

then

$$
c_{k M}^{2}=\sum_{n=2^{k-1}+1}^{\infty} \sum_{m=M}^{\infty} f_{n m}^{2} \pi
$$

Using eq.(16) to get

$$
c_{k M}^{2}<\sum_{n=2^{k-1}+1}^{\infty} \sum_{m=M}^{\infty} \pi \frac{\pi^{3} L^{2}(m+1)^{-5}}{4(m-1)^{4}}
$$

or

$$
c_{k M}^{2}<\frac{\pi^{4} L^{2}}{2^{2}} \sum_{n=2^{k-1}+1}^{\infty} \sum_{m=M}^{\infty} \frac{1}{(n+1)^{5}} \frac{1}{(m-1)^{4}}
$$

\section{Discussion}

Chebyshev wavelets of the fourth kind was presented and studied associated with some important properties. It was proved that the fourth kind Chebyshev wavelets expansion of afunction $f(x)$, with bounded second derivative, converge uniformly to $\mathrm{f}(\mathrm{x})$. An error estimation criterion formula was also introduced in this work.

\section{References}

[1] Yamg C. and Hou J., Chebyshev Wavelets Method for Solving Bratu's Problem, Yang and Hou boundary value problems, springer open journal, 2013.

[2] FariborziAraghi M. A. and Daliri S., Numerical Solution of Integro-Differential Equation by Using Chebyshev Wavelets Operational Matrix of Integration, International Journal of Mathematical Modeling \& Computations, Vol. 02, No. 02 127-136, 2012.

[3] Ali A. and Iqbal M. A., Chebyshev Wavelets Method for Delay Differential equations, International Journal of Modern Mathematical Sciences Vol. 8, No. 2, 102-110, 2013.

[4] Abd-Elhameed W. M, and Doha E. H., New Spectral Second Kind Chebyshev Wavelets Algorithm for Solving Linear and Non Linear Second -Order Differential Equations Involving Singular and Bratu Type Equations, Abstract and Applied Analysis Vol. 2013, Article ID 715756, 2013.

[5] Biazar J. and Ebraimi H.G., A Strong Method for Solving Systems of Integro-Differential Equations, Applied Mathematics, Vol.2, 1105-1113,2011.

[6] Shihab S. N. and Abdelrehman A. A., Some New Relationships Between the Derivatives of First and Second Chebyshev Wavelets, International Journal of Engineering, Business and Enterprise Application, 2012.

[7] Hosseini S. Gh., A New Operational Matrix of Derivative for Chebyshev Wavelets and its Applications in Solving Ordinary Differential Equations with Non a analytic Solution, Applied Mathematical Sciences, Vol. 5, No. 51 2537-2548, 2011.

[8] Abu Haya A. A., Solving Optimal Control Problem Via ChebyshevWavelet, Msc. Thesis, The Islmic University of Gaza, 2011,

[9] Shihab S. N, and Abdalrehman A. A., Solving Optimal Control Linear Systems by Using New Third Kind Chebyshev Wavelets Operational Matrix of Derivative, to appear.

[10] Abdalrehman A. A., Numerical Solution of Optimal Control Problems Using New Third Kind Chebyshev Wavelets Operational Matrix of Integration, to appear.

[11] Abd-Elhameed W. M, and Doha E. H., New Wavelets Collocation Method for Solving Second -Order Multipoint Boundary Value Problems Using Chebyshev Polynomials of Third and Fourth Kinds, Abstract and Applied Analysis Vol. 2013, Article ID 542839, 2013

[12] Dehghan M. and Aghigh K., A survey on Third and Fourth Kind Chebyshev Polynomials and Their Applications, http//www.ivsl.orgl?language=ar.

[13] Mason J. C. and Elliott G. H., Near- Minimax Polynomial Expansion , Journal of Computational and Applied Mathematics,http//www.ivsl.orgl?language=ar.

[14] Dehghan M. and Eslhchi M. R., The Third and Fourth Kinds of Chebyshev Polynomials and Best Uniform Approximation , mathematical and computer modeling, http//www.ivsl.orgl?language=ar. 